Annette Harth/Ulfert Herlyn/
Gitta Scheller/Wulf Tessin
Wolfsburg: Stadt am Wendepunkt 
Annette Harth/Ulfert Herlyn/

Gitta Scheller/Wulf Tessin

\section{Wolfsburg: \\ Stadt am Wendepunkt}

Eine dritte

soziologische Untersuchung

Springer Fachmedien Wiesbaden GmbH 2000 
Gedruckt auf säurefreiem und alterungsbeständigem Papier.

Die Deutsche Bibliothek - CIP-Einheitsaufnahme

\section{ISBN 978-3-8100-2661-3 ISBN 978-3-663-11888-6 (eBook)}

DOI 10.1007/978-3-663-11888-6

\section{(C) 2000 Springer Fachmedien Wiesbaden}

Ursprünglich erschienen bei Leske + Budrich, Opladen 2000

Das Werk einschließlich aller seiner Teile ist urheberrechtlich geschützt. Jede Verwertung außerhalb der engen Grenzen des Urheberrechtsgesetzes ist ohne Zustimmung des Verlages unzulässig und strafbar. Das gilt insbesondere für Vervielfältigungen, Übersetzungen, Mikroverfilmungen und die Einspeicherung und Verarbeitung in elektronischen Systemen. 


\section{Inhalt}

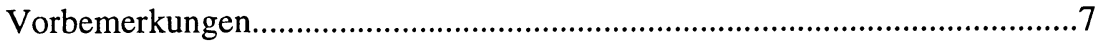

I EINFÜHRUNG:

LOKALE INTEGRATION UND MONOSTRUKTURELLE ABHÄNGIGKEIT IN WOLFSBURG .......................................... 9

$1 \quad$ Zum Forschungsstand................................................................ 11

1.1 Abriss der Entwicklung der Stadt Wolfsburg (bis 1980)................ 11

1.2 Integration in einer 'neuen Stadt' ................................................ 17

1.3 Die monostrukturelle Abhängigkeit der Stadt ............................... 23

$2 \quad$ Forschungsleitende Fragestellungen................................................ 30

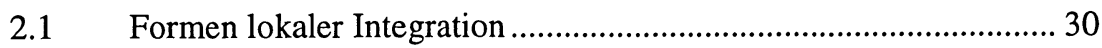

2.2 Strukturkrise und neue Kooperationsformen................................. 36

3 Methoden der Untersuchung .................................................. 40

II FORMEN LOKALER INTEGRATION ..................................... 45

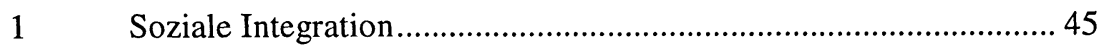

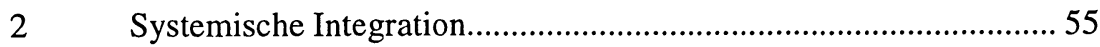

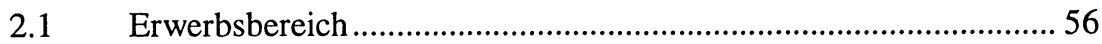

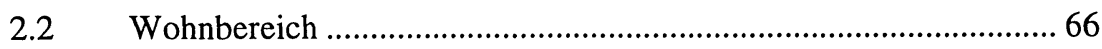

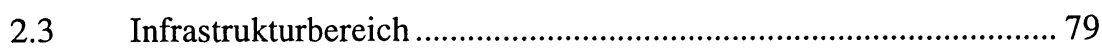

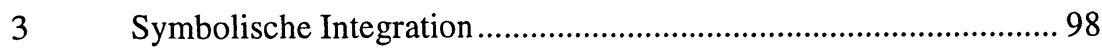

$4 \quad$ Zum Verhältnis der drei Integrationsformen ................................ 111 
III DIE VW-KRISE 1992/93 UND IHRE AUSWIRKUNGEN......... 125

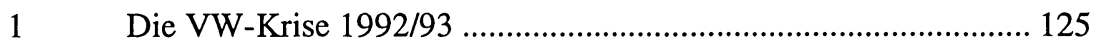

2 Die 'atmende Fabrik' und ihre Folgen ........................................... 136

2.1 Das Modell und seine Umsetzung ............................................. 136

2.2 Akzeptanz der 'atmenden Fabrik' und Vertrauen in VW ............... 143

2.3 Folgen für die Stadt und ihre Bewohnerschaft .............................. 150

3 Die VW-Krise als Wendepunkt in der Stadtentwicklung .............. 158

3.1 Die Hinwendung zum Globalisierungsregime............................. 160

3.1.1 Vom Problembewusstsein zur Aufbruchstimmung .........................160

3.1.2 Stadtentwicklung durch Großprojekte.............................................166

3.1.3 Das Leitmotiv der Erlebnisorientierung ........................................175

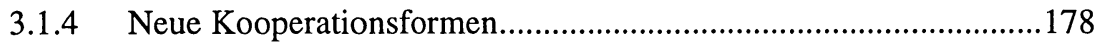

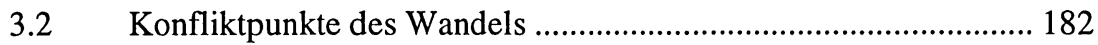

3.2.1 Zur Rolle der Bürgerschaft ............................................................183

3.2.2 Zum Verhältnis der Akteure ..................................................191

IV FAZIT:

LOKALE INTEGRATION, STRUKTURKRISE UND NEUE URBANE REGIMES .............................................................. 199

Nachwort: Ein persönlicher Rückblick auf die drei Wolfsburg-Studien (U. Herlyn)

Literaturverzeichnis 223 


\section{Vorbemerkungen}

Als die ersten Absprachen zu dieser soziologischen Untersuchung zwischen Vertretern der Stadt und uns Ende 1994 getroffen wurden, hatte die Stadt gerade die schwerste Strukturkrise im Volkswagenwerk seit seinem Bestehen mit einem Abbau von Tausenden von Arbeitsplätzen erlebt, zu deren Bewältigung ein bundesweit einmaliges Arbeitszeitmodell (die 'atmende Fabrik') entwickelt worden war. Die Stadt befand sich zwischen Hoffen auf eine Wiedererstarkung und Bangen vor einem weiteren Absturz: Sie war an einem Wendepunkt in der Stadtentwicklung angekommen. Heute, am Ende des 1997 begonnenen Forschungsprojektes wissen wir, dass die Wende für die Stadt einen Aufschwung bedeutete und ein weiterer Abschwung verhindert werden konnte.

Zentrale Themen dieser nunmehr dritten Wolfsburg-Studie sind zum einen die VW-Krise und ihre Bewältigung im Rahmen neuer Kooperationsformen zwischen Stadt und Werk, zum anderen Formen lokaler Integration auf sozialer, systemischer und symbolischer Ebene. Damit setzt diese dritte Untersuchung die Analyse zweier zentraler Themen der bisherigen soziologischen Wolfsburg-Forschung fort: die Frage der lokalen Integration in der Stadt und das Verhältnis von Stadt und VW-Werk.

Begonnen hatte die soziologische Wolfsburg-Forschung im Jahre 1959, was sich acht Jahre später in dem ersten Band niedergeschlagen hat (M. Schwonke, U. Herlyn 1967: Wolfsburg. Soziologische Analyse einer jungen Industriestadt). 20 Jahre später konnte die zweite Untersuchung der Entwicklung der Stadt durchgeführt werden (U. Herlyn, U. Schweitzer, W. Tessin, B. Lettko 1982: Stadt im Wandel. Eine Wiederholungsuntersuchung der Stadt Wolfsburg nach 20 Jahren). Gleichzeitig mit dieser dritten hier vorgelegten Arbeit veröffentlichen wir im gleichen Verlag eine historisch orientierte Gesamtdarstellung der Stadtentwicklung von Wolfsburg (U. Herlyn, W. Tessin: Faszination Wolfsburg 1938-2000).

Am Ende der ersten beiden Wolfsburg-Untersuchungen konnte man jeweils davon sprechen, dass die Stadt eine weitere Etappe in ihrer Stadtentwicklung und gemeindlichen Integration abgeschlossen hatte. Am Ende dieser dritten Studie steht Wolfsburg, mehr als 60 Jahre nach der Stadtgründung, eher am Anfang einer neuen Etappe. In zehn Jahren wird man sehen, was daraus geworden ist - der richtige Zeitpunkt für eine vierte WolfsburgStudie? 
Unser Dank gilt zunächst der Stadt Wolfsburg, die, wie zuvor schon die anderen Studien, auch dieses Projekt durch vielfältige Kooperationen und einen finanziellen Zuschuss unterstützt hat. Diese Untersuchung wurde jedoch im Wesentlichen ermöglicht durch die zweijährige Forschungsförderung der Deutschen Forschungsgemeinschaft, der wir hiermit vielmals danken.

Darüber hinaus bedanken wir uns bei:

- Frau Heidi Lüder von der Stadtentwicklung Wolfsburg, die uns während der gesamten Projektlaufzeit mit Informationen versorgt hat

- Herrn Oliver Beyer, der die elektronische Datenverarbeitung besorgt hat

- Herrn Ralph Juhnke, der als Hilfskraft über längere Zeit am Projekt mitgearbeitet hat

- Frau Corinna Haberkorn, die das Projekt verwaltungsmäßig betreut hat

- Frau Claudia Breinker, die das Layout für die Druckvorlage erstellt hat.

Im Dezember 1999

als Projektleiter

U. Herlyn und W. Tessin 